\title{
Depresión y adhesión a terapia anti-retroviral en pacientes con infección por VIH atendidos en el Hospital San Pablo de Coquimbo, Chile
}

\author{
Makarena Varela y Susan Galdames
}

\section{Depression and HAART adherence in HIV infected patients attending Hospital San Pablo of Coquimbo, Chile}

Despite the good HAART coverage of HIV infected patients in Chile, treatment success is interfered by patient-adherence problems. Moreover, HIV and depression co-morbidity has been shown to impair self-care and medication adherence. With the aim to analyze adherence problems and its relationship with depression in HIV infected patients, we evaluated 119 HIV infected patients, attending Coquimbo's Hospital. Our sample was incidental and transversally evaluated with: Morisky, Green \& Levine Medication Adherence Scale, and the Beck - II Depression Inventory. In addition, demographic and health data were obtained from clinical files. Main results showed that $68 \%$ patients referred having HAART adherence problems, with differences in sex, age and treatment duration. Logistic regression analysis showed that patients with moderate-severe depressive symptoms have 3,08 (95\% IC 1,08-8,80) times higher risk of having adherence problems compared to patients with mild to no depressive symptoms.

Key words: HIV, AIDS, HAART adherence, depression.

Palabras clave: VIH, SIDA, adhesión a TARV, depresión.

\section{Introducción}

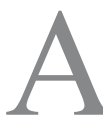

pesar de los grandes avances, la infección por VIH sigue teniendo profundas consecuencias sanitarias, demográficas y económicas para la población mundial ${ }^{1,2}$. La principal estrategia de tratamiento de esta enfermedad ha sido la implementación de la terapia anti-retroviral (TARV) en forma masiva, lo que ha permitido disminuir dramáticamente la morbimortalidad asociada a la enfermedad y ha mejorado el deterioro inmune y la calidad de vida de las personas infectadas por $\mathrm{VIH}^{3}$.

En nuestro país, desde el 2001 se tomó la decisión técnico-política de dar TARV a la población beneficiaria del Sistema de Salud Pública con recursos estatales, mediante un programa de acceso expandido a TARV ${ }^{2}$. Sin embargo, para conseguir el éxito del tratamiento con medicamentos anti-retrovirales, se requiere un alto nivel de adhesión a tratamiento en los pacientes, esto es, superior al $90 \%{ }^{4}$. El seguimiento inapropiado de la TARV puede resultar en resistencia farmacológica, la cual limita la eficacia del tratamiento, con consecuencias directas para la salud de las personas tratadas.

Es así como la adhesión al tratamiento anti-retroviral es reconocida como uno de los factores modificables de mayor relevancia para el éxito de la TARV 5 . Por esto, en la última década se han estudiado diversos factores psicosociales asociados a los problemas de adhesión a tratamiento, encontrándose factores individuales, características de la enfermedad, del sistema de salud y de la relación prestador de salud-paciente, que en su conjunto inciden en la conducta de adhesión a tratamiento por parte de los pacientes.

Entre los factores individuales que afectan la conducta de adhesión a tratamiento, se ha destacado la presencia de síntomas depresivos, los que influirían tanto en la toma de decisión para iniciar la TARV como en el proceso de adaptación y mantención del tratamiento a través del tiempo ${ }^{6}$. La evidencia hasta la fecha ha mostrado que la depresión disminuye la adhesión al tratamiento en pacientes con enfermedades crónicas en general, quienes tendrían una probabilidad dos veces mayor de no adherir a su tratamiento, por sobre quienes no están deprimidos ${ }^{7}$.

En nuestro país, se ha estimado una prevalencia para depresión mayor de $6 \%$ en las mujeres y de $3 \%$ en los hombres, a partir de los 15 años. Si se agregan los episodios de depresión leve y la distimia, la prevalencia alcanza a $10,7 \%$ entre las mujeres y $4,9 \%$ en hombres. En el caso de los pacientes infectados por VIH, la depresión suele ser dos a cuatro veces más frecuente que en la población general (alrededor de 37\%), considerándose como una de las co-morbilidades psiquiátricas más prevalentes y de mayor incidencia tras el diagnóstico de la infección $^{8}$. Un estudio reciente, que evaluó características sociodemográficas y síntomas de pacientes con VIH, encontró que la depresión -como síntoma- se encontraba
Universidad de La Serena, La Serena, Chile.

Departamento de Psicología (SGC, MV).

Hospital San Pablo de Coquimbo. Chile. Unidad de VIH/SIDA (MV).

Las autoras declaran no tener conflictos de interés con institución alguna.

El estudio fue financiado por recursos generados por sus autoras principales.

Recibido: 18 de julio de 2013 Aceptado: 7 de abril de 2014

Correspondencia a: Makarena Varela makarena.varela@redsalud.gov.cl sgaldame@userena.cl 
en $5^{\circ}$ lugar entre los síntomas más reportados (46\%) por los pacientes 9 .

La depresión y los problemas de salud mental se han asociado con adhesión a la TARV en forma directa y, además, se han reconocido como mediadores del efecto del apoyo social sobre la adhesión a TARV ${ }^{10}$. Según esto último, el efecto que puede tener la red de apoyo social sobre la adhesión a tratamiento dependería de la salud mental de los pacientes.

Por otro lado, se ha planteado que la infección por VIH/SIDA aumenta la probabilidad de tener depresión como resultado de múltiples estresores que complican la vida de las personas infectadas con el virus, tal como la reducción del apoyo social, el aislamiento y los malos tratos, así como el ajuste a complicados regímenes de medicamentos antirretrovirales ${ }^{8}$.

Entonces, la co-morbilidad entre VIH y depresión se constituye en un peor manejo del curso de la infección por $\mathrm{VIH}$, dado que esta última puede afectar negativamente la evolución de la enfermedad, con peor estado inmunológico expresado en un menor recuento de linfocitos $\mathrm{T}$ CD4+, mayor carga viral, progresión de la enfermedad, deterioro de la calidad de vida, mayor morbi-mortalidad y consecuente uso del sistema de salud.

Una de las formas en que la depresión agravaría el manejo de la infección por VIH/SIDA, a nivel conductual, sería a través de empeorar la adhesión a los medicamen$\operatorname{tos}^{5,11,8}$

En base a los antecedentes revisados, la presente investigación se propuso evaluar la presencia de síntomas depresivos y problemas de adhesión a TARV, y la relación entre ambos, en pacientes que se encuentran bajo control en el Programa VIH/SIDA del Hospital San Pablo de Coquimbo. Se estudiaron estas variables desde un enfoque de riesgo, de acuerdo al cual se hace necesario conocer la presencia de co-morbilidad y problemas psicológicos que obstaculizan el éxito de la TARV y disminuyen la calidad de vida de los pacientes. Además, se caracterizaron algunos factores psicosociales de pacientes adherentes y no adherentes ${ }^{5,12}$

\section{Material y Método}

El diseño de investigación que comprende este estudio es de tipo descriptivo relacional, transeccional ${ }^{13}$, con muestreo incidental. De este modo, se evaluó una muestra conformada por 119 sujetos, correspondientes a $80 \%$ del total de los pacientes atendidos en el Programa VIH/SIDA del Hospital San Pablo de Coquimbo. Los criterios de inclusión fueron haber recibido TARV al menos durante tres meses y aceptar voluntariamente participar en la investigación. Se excluyó a quienes tenían atención psicoterapéutica al momento de la evaluación

\begin{tabular}{|c|c|c|}
\hline & Promedio \pm DS & Rango \\
\hline Edad (años) & $42,89 \pm 10,92$ & $19-69$ \\
\hline Tiempo de tratamiento (años) & 6,41 & $1-16$ \\
\hline Recuento de $\mathrm{CD} 4 / \mathrm{mm}^{3}$ & $450,77 \pm 211,56$ & $62-1.093$ \\
\hline $\mathrm{n}$ pastillas & $3,68 \pm 1,68$ & $2-12$ \\
\hline
\end{tabular}

y quienes tenían diagnóstico de deterioro neurológico o trastorno psiquiátrico previo. Un $76 \%$ de la muestra estuvo conformada por hombres y $24 \%$ por mujeres (Tabla 1 ).

En una primera fase de la investigación se realizó un pilotaje de los instrumentos, con la finalidad de probar su aplicabilidad y aceptación por parte de los pacientes. Luego se realizó la selección y evaluación de los sujetos, quienes en su visita a control o retiro de fármacos, fueron entrevistados en dependencias del Centro de Atención Integral del Hospital San Pablo de Coquimbo y, posteriormente, se accedió a información registrada en las historias clínicas de cada paciente.

Los análisis fueron hechos con el programa SPSS (Statistical Package for the Social Sciences) en su versión 20.0, con un nivel de significación de 0,05. El análisis comenzó con la descripción de medidas de tendencia central y frecuencia. Luego, un análisis de diferencias con $\chi^{2}$ para variables categoriales y $t$ de student o ANOVA para las variables contínuas. Finalmente, se calculó una regresión logística que permite observar la relación entre las variables estudiadas que habían mostrado una relación significativa. Debido a que el diseño transversal de este estudio no permite identificar relaciones causales entre las variables, la estimación de los odds ratio se usó como estimador de riesgo y no como predictor.

El proyecto contó con la aprobación del Comité de Ética hospitalario y todos los procedimientos utilizados se ajustaron a los criterios estándares requeridos en una investigación con personas (Declaración de Helsinki 2004).

\section{Instrumentos}

Para medir adhesión a tratamiento farmacológico utilizamos el Test de Morisky-Green-Levine ${ }^{14}$, consistente en un cuestionario de auto-reporte de cuatro preguntas con respuesta dicotómica, donde el sujeto responde sí/no, de acuerdo a su conducta frente a la TARV. Se considera adherente al paciente que contesta afirmativamente a las cuatro preguntas, teniendo adherencia óptima, mientras que a partir de una respuesta negativa se califica como no adherente. Este instrumento ha sido usado en la valoración de la adhesión al tratamiento en diferentes patologías como la hipertensión arterial, esquizofrenia, diabetes mellitus, cáncer, cardiopatía, osteoporosis, enfermedad 
tromboembólica, patología infecciosa, tabaquismo, dislipemia, EPOC y asma bronquial ${ }^{15}$. Algunos estudios indican que el test de Morisky-Green-Levine tiene un valor predictor positivo, por lo cual sería una buena alternativa para identificar grupos de riesgo de no adherir al tratamiento medicamentoso ${ }^{15}$. Por otra parte, un estudio que comparó una escala de auto-reporte de adhesión con el monitoreo sanguíneo de los medicamentos, concluyó que la medición de auto-reporte es un instrumento bastante preciso y confiable para medir adhesión al tratamiento en pacientes con infección por VIH/SIDA ${ }^{16}$.

La variable depresión fue medida con el Inventario de Depresión de Beck-II, el cual es un instrumento de autoreporte tipo escala lickert, compuesto por 21 ítems cuya finalidad es medir el grado de intensidad de los síntomas depresivos. El sujeto puntúa de 0 a 3 según la elección de la frase que más lo representa en cada ítem propuesto. La adaptación española del inventario ${ }^{17}$ mostró una consistencia interna elevada (Alpha de Cronbach: 0,89). En esa versión se encontró que el BDI-II mide una dimensión general de depresión compuesta por dos factores: uno cognitivo-afectivo y otro somático-motivacional. Los ítems del BDI-II cubren todos los criterios diagnósticos del DSM-IV para depresión, lo cual respalda su validez de contenido; sin embargo, cabe mencionar que para especificar un diagnóstico clínico los autores recomiendan la evaluación de un profesional debidamente acreditado.

De igual manera, en Argentina se hizo una adaptación del BDI-II al castellano ${ }^{18}$, la cual mostró una adecuada consistencia interna (Alpha de Cronbach: 0,88-0,86) y estabilidad en el tiempo (correlación test-retest de 7-10 días, r: 0,90). Los puntos de corte que se establecieron clasifican la puntuación total en las siguientes categorías: 0-13: depresión mínima; 14-19: depresión leve; 20-28: depresión moderada, y 29-63: depresión grave.

Además de las variables adhesión a tratamiento y depresión, a partir de las fichas clínicas de los pacientes se registraron variables sociodemográficas y clínicas (Tabla 2).

\section{Resultados}

La prevalencia de depresión medida con el BDI-II tuvo un promedio de $14,30 \pm 12,2$, fluctuando entre $0 \mathrm{y}$ 59 puntos. En términos de las categorías correspondientes a los puntajes obtenidos se observó $59 \%$ de casos con depresión mínima, 13,1\% con depresión leve, 16,4\% depresión moderada y $11,5 \%$ depresión severa. Para describir los síntomas depresivos específicos prevalentes entre los pacientes evaluados, calculamos el porcentaje de sujetos cuya respuesta indicaba la presencia de cada síntoma, sin considerar su intensidad. De este modo, observamos que alrededor de $50 \%$ de los pacientes manifestó
Tabla 2. Características socio-demográficas de la muestra

\begin{tabular}{lcc|} 
& Frecuencia & $\%$ \\
Sexo & & \\
$\quad$ Hombre & 92 & 75,4 \\
Mujer & 28 & 23,0 \\
Educación & & \\
$\quad$ Básica & 27 & 22,1 \\
Media & 43 & 35,2 \\
Técnica & 26 & 21,3 \\
Universitaria & 23 & 18,9 \\
Estado civil & & \\
Soltero & 67 & 55,8 \\
Casado/convive & 38 & 31,1 \\
Viudo & 4 & 3,3 \\
Separado/divorciado & 11 & 9,2 \\
Trabaja actualmente & & \\
Sí & & \\
No & 72 & 59,0 \\
Apoyo social percibido & 47 & 38,5 \\
Suficiente & & \\
Insuficiente & 91 & 74,6 \\
\hline
\end{tabular}

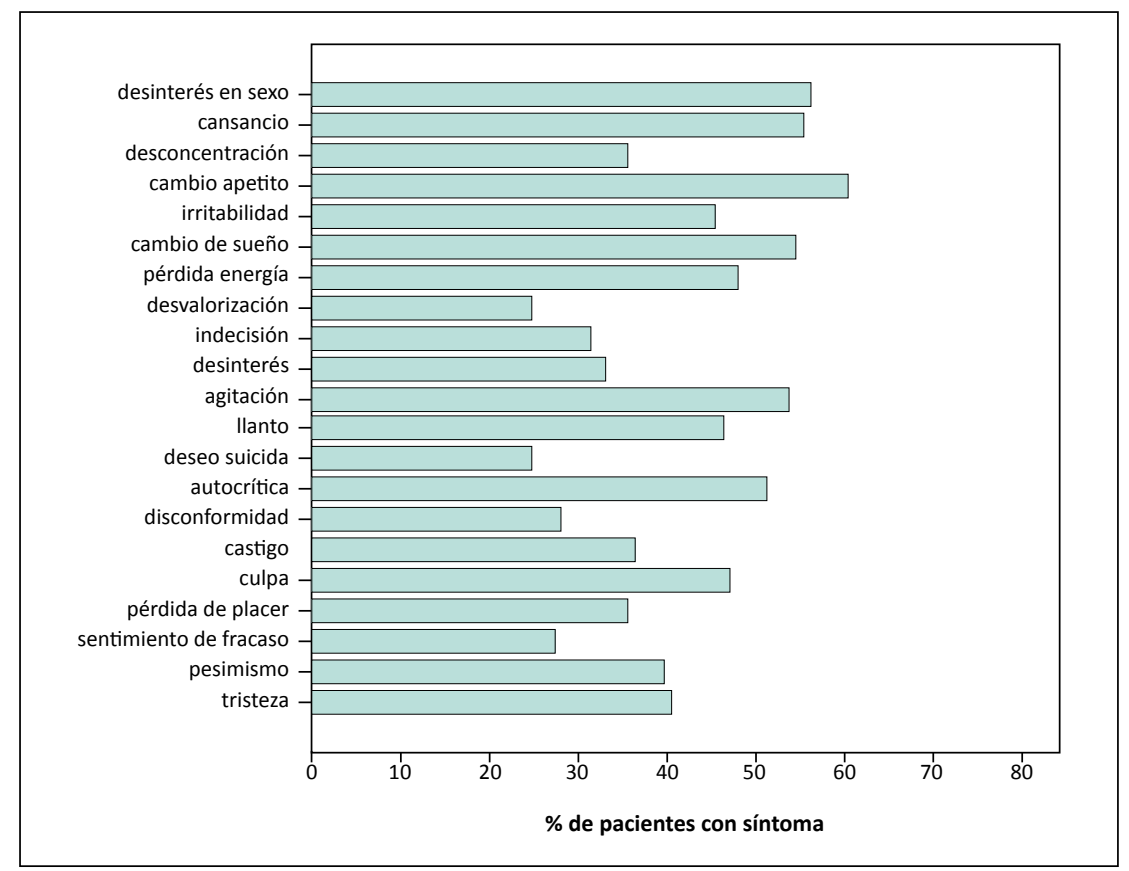

Figura 1. Síntomas depresivos referidos en 120 pacientes en control por infección VIH/SIDA en el Hospital San Pablo, Coquimbo.

experimentar: cambio de apetito, pérdida de interés en el sexo, cansancio, cambios en el sueño, agitación, autocrítica y culpa. Los síntomas presentes en menos de 30\% de los pacientes fueron: desvalorización, deseo suicida, disconformidad y sentimiento de fracaso (Figura 1). 


\begin{tabular}{|c|c|c|c|c|c|c|c|c|}
\hline & \multirow[b]{2}{*}{$\mathbf{n}$} & \multicolumn{3}{|c|}{ Sintomatología depresiva } & \multicolumn{4}{|c|}{ Adherencia a TARV } \\
\hline & & Promedio \pm DS & Prueba $t$ & Signif. & $\begin{array}{l}\text { Adherentes } \\
\text { (n) }\end{array}$ & $\begin{array}{l}\text { No adherentes } \\
\text { (n) }\end{array}$ & $x^{2}$ & Signif. \\
\hline Hombres & 92 & $12,2 \pm 10,91$ & $2,61_{(1,37.3)}$ & $0,013^{*}$ & 33 & 59 & 4,68 & 0,03 \\
\hline Mujeres & 28 & $19,8 \pm 14,14$ & & & 4 & 24 & & \\
\hline Total & 120 & & & & 37 & 83 & & \\
\hline
\end{tabular}

Los análisis psicométricos del BDI-II mostraron un índice de confiabilidad alta (alpha de Chronbach: 0,91), con correlaciones ítem test en el rango de 0,36-0,67. La presencia de depresión mostró diferencias por sexo ( $\mathrm{t}$ de student), donde las mujeres tenían mayor severidad de la depresión que los hombres (Tabla 1).

La adhesión auto-reportada a TARV, medida en forma dicotómica, mostró $30,3 \%$ de sujetos considerados óptimamente adherentes y $68,0 \%$ no adherentes. Además, se observó diferencia por sexo según la cual habría un mayor número de mujeres que no adhieren de acuerdo a lo estadísticamente esperado (Tabla 3).

Tabla 4. Diferencia en años de tratamiento de pacientes adherentes versus no adherentes a TARV

\begin{tabular}{|lrccccccc|} 
& n & Promedio & DS & Mínimo & Máximo & F & gl & Sig. \\
Adherentes & 36 & 5,17 & 3,97 & 0,2 & 12,4 & 4,83 & 1 & 0,03 \\
No-adherentes & 81 & 7,04 & 4,36 & 0,3 & 16,3 & & & \\
Total & 117 & 6,46 & 4,32 & 0,2 & 16,3 & & 116 \\
\hline
\end{tabular}

Figura 2. Adherencia a TARV según edad del paciente.
Otra variable que mostró diferencia entre pacientes adherentes y no adherentes fue la edad. Según un ANOVA, el promedio de edad de los pacientes no adherentes $(41,4$ $\pm 14,7)$ fue significativamente más bajo que el promedio de los adherentes $(46,08 \pm 10,7)$ ( $\left.F: 4,70_{1,119}, \mathrm{p}: 0,032\right)$. Tras esto analizamos los rangos de edad de los pacientes y observamos que es posterior a los 40 años que la proporción entre adherentes vs no adherentes cambia siendo mayor la proporción de pacientes adherentes (Figura 2).

Se encontró diferencia en el tiempo de tratamiento entre pacientes adherentes y no adherentes, donde los pacientes adherentes tuvieron una media significativamente menor de años de TARV que los no adherentes (Tabla 4).

Las variables: estado civil, trabajo, nivel educacional y apoyo percibido no mostraron diferencias significativas entre pacientes adherentes y no adherentes (Tabla 2).

\section{Relación entre adhesión a TARV y depresión}

Un análisis de regresión logística binaria permitió determinar en qué medida la intensidad de la depresión es un factor de riesgo significativo para la adhesión vs no adhesión en los sujetos.

Para realizar los análisis se dicotomizó la variable depresión usando el punto de corte de 20 del BDI-II. De este modo, las categorías resultantes fueron: depresión mínima o leve (0) y depresión moderada o severa (1). Esta variable se introdujo como predictor de la variable dependiente: adherente (0) v/s no-adherente (1). El modelo predijo correctamente $69 \%$ de casos. Según este modelo, las personas con depresión moderada o severa tienen una probabilidad tres veces superior de ser no adherentes que los pacientes con depresión mínima o leve $(3,08)$.

En un segundo modelo ingresamos las variables que previamente habían mostrado resultados significativos sobre adhesión: edad, tiempo de tratamiento y sexo. Sin embargo, dado que la variable sexo no resultó significativa en presencia de los otros predictores fue sacada del modelo final. El segundo modelo permitió predecir correctamente $68 \%$ de los casos (Tabla 5). 


\begin{tabular}{|c|c|c|c|c|c|c|c|c|c|c|c|}
\hline \multicolumn{2}{|c|}{ Modelo } & B & Wald & gl & Sig. & $\operatorname{Exp}(B)$ & \multicolumn{2}{|c|}{ I.C. $95 \%$ para EXP(B) } & $\chi^{2}$ & Sig. & $\begin{array}{c}-2 \text { log de la } \\
\text { verosimilitud }\end{array}$ \\
\hline \multirow{2}{*}{$1^{\circ}$} & Síntomas donrociva & 1127 & 1137 & 1 & 003 & 308 & 108 & 880 & $5160(1)$ & 00073 & בח1 19 \\
\hline & Constante & 0,560 & 6,377 & 1 & 0,01 & $\begin{array}{l}5,70 \\
1,75\end{array}$ & 1,00 & 0,00 & $5,100(1)$ & , & 140,102 \\
\hline \multirow[t]{4}{*}{$2^{\circ}$} & Edad & $-0,046$ & 5,073 & 1 & 0,02 & 0,95 & 0,91 & 0,99 & $15,63(3)$ & 0,001 & 128,800 \\
\hline & Años de TARV & 0,135 & 6,568 & 1 & 0,01 & 1,14 & 1,03 & 1,27 & & & \\
\hline & Síntomas depresivos & 1,127 & 4,106 & 1 & 0,04 & 3,08 & 1,03 & 9,18 & & & \\
\hline & Constante & 1,734 & 3,580 & 1 & 0,05 & 5,66 & & & & & \\
\hline
\end{tabular}

\section{Discusión}

La prevalencia de pacientes con infección por VIH/ SIDA y depresión moderada o severa fue de $28 \%$, lo que es superior a lo observado en población chilena y se encuentra en los rangos señalados por estudios en pacientes seropositivos. Los principales síntomas declarados fueron de carácter somático, los cuales podrían asociarse o no al proceso biológico de enfermedad, lo que es una discusión interesante y que supera los objetivos del presente estudio.

Un $68 \%$ de los pacientes reportó problemas de adhesión a TARV, lo cual subraya la necesidad de comprender mejor esta problemática y delinear estrategias efectivas que permitan intervenir sobre el problema. Nuestro estudio encontró que los pacientes con depresión moderadasevera tienen una probabilidad tres veces mayor de ser no-adherentes respecto a los que tienen depresión mínima o leve. Conforme a esto, es relevante considerar el manejo de la co-morbilidad infección por VIH/SIDA-depresión no sólo como una forma de mejorar la calidad de vida, a nivel psicológico, de los pacientes sino que de aumentar los niveles de adhesión a TARV. Esto supone la capacitación integral de los equipos de salud en la detección y abordaje de los pacientes con depresión y no sólo su manejo terapéutico por parte del profesional psicólogo. En este sentido, entre las aproximaciones al problema de la depresión en servicios de salud, un estudio reciente mostró que los proveedores de salud muestran actitudes negativas hacia los pacientes con depresión, dado que presentan altas necesidades emocionales. Los pacientes tendieron a estar más descontentos con la atención recibida y en general se observó que la comunicación proveedor-paciente fue más difícil ${ }^{19}$. Creemos que se requiere capacitar a los equipos en estrategias de manejo especializadas para tratar con estos pacientes.

Otro resultado interesante fue la asociación entre adhesión a TARV y edad de los pacientes. Los pacientes bajo 40 años de edad mostraron más problemas de adhesión que los mayores. Esto es consistente con otros estudios de adhesión al tratamiento que muestran una mejor adhesión en pacientes de mayor edad ${ }^{20,21}$. Nuestra hipótesis al res- pecto, es que los pacientes de menor edad tienen menor percepción de vulnerabilidad, por sus características evolutivas; sin embargo, es un factor que sería interesante esclarecer en futuras investigaciones.

Por otra parte, se observó que después de seis años de tratamiento aumentaban los problemas de adhesión en los pacientes. Esto nos permite plantear la hipótesis de un curso de adhesión a TARV variable en el tiempo, lo que también queda por investigar. De todos modos descartamos que el tiempo de tratamiento se asociara al efecto de la edad en la adhesión al tratamiento, ya que ambas variables no mostraron estar asociadas en esta muestra, es decir, los pacientes de mayor edad no necesariamente tenían más años de tratamiento.

A pesar de que evaluamos una menor proporción de mujeres, en ellas observamos mayor intensidad de la depresión, lo cual es esperable según la mayor prevalencia general de depresión en mujeres; sin embargo, a esto se sumó que tienen peor adhesión a TARV, lo cual es coherente con otros estudios ${ }^{20,22,23}$. De este modo, ser mujer e infectada con VIH se asocia a mayor riesgo en salud y, posiblemente, menor éxito de la TARV y peor calidad de vida. Consideramos relevante que en futuras investigaciones se profundice en las motivaciones y factores que impactan la adhesión al tratamiento según un enfoque de género, ya que es un ámbito con escasa investigación en la actualidad ${ }^{24}$.

Por último, el desafío propuesto por la OMS en torno a proveer de un cuidado comprensivo y apoyo a las personas infectados con VIH, implica considerar la prevalencia de depresión y su efecto en la adhesión a TARV como un problema a tratar en forma integral. La evidencia en torno a intervenciones para mejorar adhesión a tratamiento sugiere que los factores relacionales paciente-equipo de salud y los factores cognitivos del paciente son puntos clave a fortalecer ${ }^{25,26}$. Nuestros hallazgos nos invitan a reflexionar sobre cómo estos factores están interferidos por los síntomas depresivos de los pacientes ${ }^{27}$, y cómo se podrían establecer medidas de abordaje integral por parte de los equipos de salud. En este sentido la creciente disciplina de la Psicología de la Salud en Chile puede ofrecer aportes ${ }^{28}$. 


\section{Resumen}

A pesar de la cobertura de TARV en pacientes infectados con VIH lograda en Chile, el éxito de los tratamientos se encuentra interferido por problemas de adhesión de los pacientes. Además, la co-morbilidad entre infección por VIH y depresión ha mostrado empeorar la adhesión y el automanejo de las enfermedades crónicas en general. Con el objetivo de analizar los problemas de adhesión a TARV y su relación con la depresión en pacientes infectados con VIH, se evaluó a 119 pacientes atendidos en el Programa VIH/SIDA del Hospital de Coquimbo. La muestra fue in- cidental y fueron aplicados en forma transversal: la Escala de Adhesión a los Medicamentos de Morisky, Green y Levine, y el Inventario de Depresión de Beck-II. Además se obtuvieron datos demográficos y de salud de las fichas clínicas. Los principales resultados muestran que $68 \%$ de los pacientes manifiesta tener problemas de adhesión a TARV, con diferencias significativas por sexo, edad y tiempo de tratamiento. Según un análisis de regresión logística, los pacientes con depresión moderada a severa tendrían 3,08 (95\% IC 1,08-8,80) veces más riesgo de tener problemas de adhesión en relación a los pacientes con depresión mínima o leve.

\section{Referencias bibliográficas}

1.- Ministerio de Salud. Evaluación del impacto de una política pública: Ampliación del acceso a terapia antirretroviral en Chile. Rev Chilena Infectol 2003; 20 (4): 277-84.

2.- Organización Mundial de la Salud. Objetivos de Desarrollo del Milenio: Informe de 2011. Nueva York: OMS 2011

3.- Beltrán C, Wolff M, Gallardo D, Arancibia J, Morales O. Evaluación de impacto del programa de acceso expandido a tratamiento antirretroviral cohorte chilena de SIDA. Santiago de Chile: Ministerio de Salud, Comisión Nacional del SIDA 2007.

4.- Marco A, Knobel H, Guelar A. La adhesión al tratamiento antirretroviral; una perspectiva global del problema y posibles soluciones. Enf Emerg 2000; 2 (1): 45-53.

5.- Organización Mundial de la Salud. Adherencia a los tratamientos a largo plazo: Pruebas para la acción. Ginebra: OMS; 2004.

6.- Xochihua-Díaz L. Apego al tratamiento antirretroviral en adolescentes. Bol Med Hosp Infant Mex 2009; 66 (4): 306-13.

7.- Nogueira L, Crosland M D, Remien R H. Anxiety and depression symptoms as risk factors for non-adherence to antiretroviral therapy in Brazil. AIDS Behav 2008; 14 (2): 289-99.

8.- Daughters S, Magidson J, Schuster R, Safren S. ACT HEALTHY: A combined cognitive-behavioral depression and medication adherence treatment for HIV-infected substance users. Cogn Behav Pract 2010; 17 (3): 309-21.

9.- Araya A X, Norr K F, Pérez C M, Levy J A, Park C G, Kim M J. HIV-related symptoms and patient clusters among Chileans living with HIV. AIDS Care 2012; 22 (3): 355-60.

10.- Huynh A K, Kinsler J J, Cunningham W E, Sayles J N. The role of mental health in mediating the relationship between social support and optimal ART adherence. AIDS Care 2013; 22 (3): 355-60.
11.- Ministerio de Salud Pública. La adherencia al tratamiento antirretroviral en personas con VIH: Información para el equipo de salud. Programa nacional de prevención y control de las ITS/ VIH/SIDA, Dirección nacional de asistencia médica. Cuba: MINSAP; 2009.

12.- Wolff C, Alvarado R, Wolff M. Prevalencia, factores de riesgo y manejo de la depresión en pacientes con infección por VIH: Revisión de la literatura. Rev Chilena Infectol 2010; 27 (1): 65-7.

13.- Hernández R, Fernández-Collado C, Baptista P. Metodología de la investigación. $4^{\mathrm{a}}$ ed. México DF: Mc Graw Hill; 2006; 45-518.

14.- Morisky D, Green L, Levine D. Concurrent and predictive validity of a self-reported measure of medication adherence. Med Care 1986; 24 67-74.

15.- Rodríguez M. Efecto de la actuación farmacéutica en la adherencia del tratamiento farmacológico de pacientes ambulatorios con riesgo cardiovascular. Tesis para optar al grado de doctor, Granada: Universidad de Granada; 2008.

16.- Bulgiba A, Mohammed UY, Chik Z, Lee C, Peramalah D. How well does self-reported adherence fare compared to therapeutic drug monitoring in HAART? Prev Med 2013; 22 (3) 355-60.

17.- Sanz J, Navarro M, Vásquez C. Adaptación española del Inventario para la Depresión de Beck-II (BDI-II): Propiedades psicométricas en estudiantes universitarios. An y Mod Cond 2003; 29 (124): 239-88.

18.- Beck A, Sterr R A, Brown G K. Manual de Inventario de Depresión de Beck BDI II. Adaptación Argentina. Buenos Aires: Paidós; 2006.

19.- Jonassaint C R, Haywood C Jr, Korthuis P T, Cooper L A, Saha S, Sharp V, et al. The impact of depressive symptoms on patient-provider communication in HIV care. AIDS Care 2013; 22 (3): 355-60
20.- Knobel H, Polo R, Escobar I. Recomendaciones GESIDA/SEFH/PNS para mejorar la adherencia al tratamiento antirretroviral (Actualización). Farm Hosp 2008; 23 (4): 215-29.

21.- Alvis O, De Coll L, Chumbimune L, Díaz C, Díaz J, Reyes M. Factores asociados a la no adherencia al tratamiento antirretroviral de gran actividad en adultos infectados con el VIHSIDA. An Fac Med 2009; 70 (4): 266-72.

22.- Antakly de Mello V, Malgebier A. Depression in women infected with HIV. Rev Bras Psiquiatr 2006; 28: 10-7.

23.- Watt M H, Maman S, Golin C E, Earp J A, Eng E, Bangdiwala S I, et al. Factors associated with self-reported adherence to antirretroviral therapy in a Tanzanian setting. AIDS Care 2010; 22 (3): 381-9.

24.- Fitzgerald M, Collumbien M, Hosegood V. "No one can ask me "Why do you take that stuff?'": men's experiences of antiretroviral treatment in South Africa. AIDS Care 2010; 22 (3): 355-60.

25.- Herrera C, Campero L, Caballero M, Kendall T. Relación entre médicos y pacientes con VIH: influencia en apego terapéutico y calidad de vida. Rev Saude Publica 2008; 42 (2): 249-55

26.- González J S, Batchelder A W, Psaros C, Safren S A. Depression and HIV/AIDS treatment nonadherence. A review and metaanalysis. J Acquir Immune Defic Syndr 2011 58 (2): 181-7.

27.- Detweiller-Bedell J, Friedman M, Leventhal H, Miller I, Leventhal E. Integrating co-morbid depression and chronic physical disease management. Identifing and resolving failures in self-regulation. Clin Psychol Rev 2008; 28 (8): 1426-46.

28.- Galdames S. Validación preliminar de una intervención psicosocial breve para aumentar adherencia y disminuir síntomas depresivos en personas con hipertensión descompensada y síntomas depresivos. Tesis para optar al grado de Doctor en Psicología. 2013. PUC. 